\title{
Pirfenidone alleviates lung ischemia-reperfusion injury in a rat model
}

\author{
Masao Saito, MD, ${ }^{a}$ Toyofumi F. Chen-Yoshikawa, MD, PhD, ${ }^{a}$ Kimitaka Suetsugu, BS, ${ }^{b}$ Ryo Okabe, MD, \\ Akihiro Takahagi, MD, ${ }^{\mathrm{a}}$ Satohiro Masuda, $\mathrm{PhD},{ }^{\mathrm{b}}$ and Hiroshi Date, $\mathrm{MD}, \mathrm{PhD}^{\mathrm{a}}$
}

\section{ABSTRACT}

Objective: Lung ischemia-reperfusion injury is among the complications seen after lung transplantation, resulting in morbidity and mortality. Pirfenidone, an antifibrotic agent for the treatment of idiopathic pulmonary fibrosis, is reported to have cytoprotective properties in various disease models. The purpose of this study was to investigate the effect of pirfenidone on lung ischemia-reperfusion injury.

Methods: Male Lewis rats (260-290 g) were divided into 3 groups: sham group $(\mathrm{n}=5)$, warm ischemia $(\mathrm{WI})$ group $(\mathrm{n}=10)$, and WI plus pirfenidone (WI+PFD) group $(n=10)$. The sham group underwent 210 minutes of perfusion without ischemia. The WI and WI+PFD groups underwent 90 minutes of warm ischemia and 120 minutes of reperfusion. In the WI+PFD group, pirfenidone $(300 \mathrm{mg} / \mathrm{kg})$ was administered orally by gavage 30 minutes before ischemia. After reperfusion, arterial blood gas analysis, lung mechanics, lung wet-to-dry weight ratio, and histologic findings were obtained. The gene expressions of proinflammatory cytokines in lung tissue were measured by quantitative reverse transcription polymerase chain reaction.

Results: Compared with the WI group, the WI+PFD group had significantly better dynamic pulmonary compliance $(P<.01)$ and oxygenation levels $(P<.05)$. The wet-to-dry ratio was lower in the WI+PFD group $(P<.05)$. Histologic analysis showed that the WI+PFD group had reduced perivascular edema and neutrophil infiltration. The expression of tumor necrosis factor- $\alpha$ messenger RNA was decreased in the WI+PFD group $(P<.05)$.

Conclusions: Our results revealed that in a rat hilar clamp model, pirfenidone alleviated lung ischemia-reperfusion through anti-inflammatory effects. ( $\mathrm{J}$ Thorac Cardiovasc Surg 2019;158:289-96)

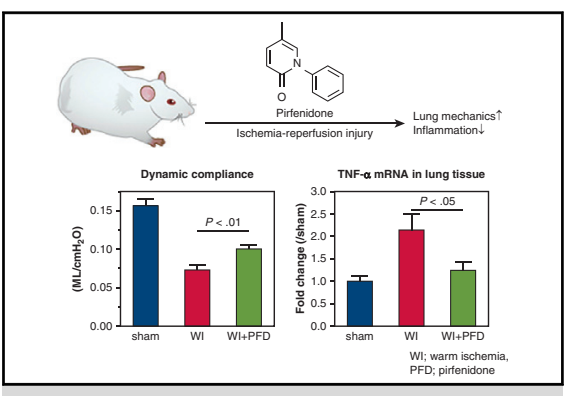

Pirfenidone alleviates lung ischemia-reperfusion injury in a rat model.

\section{Central Message}

Pirfenidone attenuated deterioration of pulmonary function by suppressing inflammation in a rat model of lung ischemia-reperfusion injury.

\section{Perspective}

Administration of pirfenidone before ischemiareperfusion injury alleviated lung edema and inflammation in the acute phase. Pirfenidone may be useful as a new option for treatment of lung ischemia-reperfusion injury.

See Commentaries on pages 297 and 299.

Lung ischemia-reperfusion injury (IRI) is characterized by nonspecific alveolar damage, lung edema, and hypoxemia that can occur in several clinical situations, such as lung transplantation, ${ }^{1-3}$ pulmonary embolism, ${ }^{4}$ cardiopulmonary bypass cardiac surgery, ${ }^{5}$ single-lung ventilation, ${ }^{6}$ and high-volume resuscitation. ${ }^{7}$ In particular, in lung

\footnotetext{
From the ${ }^{a}$ Department of Thoracic Surgery, Graduate School of Medicine, Kyoto University, Kyoto, Japan; and ${ }^{\mathrm{b}}$ Department of Pharmacy, Kyushu University Hospital, Fukuoka, Japan.

Pirfenidone was provided by Shionogi \& Co. Ltd.

Received for publication May 7, 2018; revisions received Aug 27, 2018; accepted for publication Aug 29, 2018; available ahead of print Oct 29, 2018

Address for reprints: Hiroshi Date, MD, PhD, Department of Thoracic Surgery, Graduate School of Medicine, Kyoto University, 54 Shogoin-Kawahara-cho, Sakyo-ku, Kyoto 606-8507, Japan (E-mail: hdate@kuhp.kyoto-u.ac.jp). $0022-5223 / \$ 36.00$

Copyright (C) 2018 by The American Association for Thoracic Surgery https://doi.org/10.1016/j.jtcvs.2018.08.098
}

transplantation IRI is the main cause of primary graft dysfunction, which is a major cause of mortality and morbidity in the postoperative period. ${ }^{8}$ Furthermore, lung IRI is associated with an increased risk of chronic lung allograft dysfunction. ${ }^{9}$ It is thus important to alleviate lung IRI to improve survival after lung transplantation.

Pirfenidone is an anti-fibrotic agent used for idiopathic pulmonary fibrosis (IPF) and IPF is one of the diseases for which lung transplantation is performed. ${ }^{10}$ For this reason, pirfenidone is often taken orally by patients with

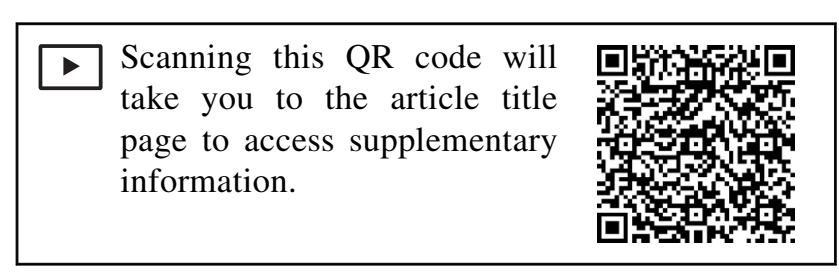




$\begin{array}{ll}\text { Abbreviations and Acronyms } \\ \text { IRI } \quad=\text { ischemia-reperfusion injury } \\ \text { IPF } & =\text { idiopathic pulmonary fibrosis } \\ \text { WI } & =\text { warm ischemia } \\ \text { WI }+ \text { PFD } & =\text { warm ischemia plus pirfenidone } \\ \text { ssDNA } & =\text { single-strand DNA } \\ \text { HPF } & =\text { high-power field } \\ \text { PCR } & =\text { polymerase chain reaction } \\ \text { TNF- } \alpha & =\text { tumor necrosis factor } \alpha \\ \text { mRNA } & =\text { messenger RNA } \\ \text { IL-1 } \beta & =\text { interleukin- } 1 \beta \\ \text { NF- } \kappa \text { B } & =\text { nuclear factor subunit } \kappa \text { B } 1 \\ \text { cDNA } & =\text { complementary DNA } \\ \end{array}$

severe IPF before transplantation. The potential antiinflammatory role of pirfenidone has been well documented in various studies. ${ }^{11,12}$ In addition, it has been already reported that pirfenidone attenuates IRI in other organs (small intestine ${ }^{13}$ and liver ${ }^{14}$ ). The effect of pirfenidone on lung IRI, however, is unknown. The purpose of this study was to investigate the effect of pirfenidone on lung IRI in a rat model.

\section{MATERIALS AND METHODS}

This study consisted of 2 experiments (experiment 1 and experiment 2). Experiment 1 was intended to investigate the function and inflammatory change in the lung after ischemia-reperfusion injury. Experiment 2 was intended to investigate concentrations of pirfenidone in blood and lung tissue.

\section{Animals}

All experimental protocols received approval from the Ethics Committee of the Graduate School of Medicine at Kyoto University. We used male Lewis rats, 11 weeks old and weighing 260 to $290 \mathrm{~g}$ (Japan SLC, Hamamatsu, Japan).

\section{Chemicals}

Pirfenidone for administration to the animals was provided by Shionogi \& Co., Ltd. (Osaka, Japan). Pirfenidone for concentration measurement was purchased from Tokyo Chemical Industry Co Ltd (Tokyo, Japan), and carbamazepine was purchased from Sigma-Aldrich Co (St Louis, Mo). All other chemicals were of the highest purity available.

\section{Experiment 1}

Rat left hilar clamp model. Rats were randomly assigned to 3 groups: sham group $(\mathrm{n}=5)$, warm ischemia (WI) group $(\mathrm{n}=10)$, and WI plus pirfenidone (WI+PFD) group $(\mathrm{n}=10)$. Pirfenidone $(300 \mathrm{mg} / \mathrm{kg})$ dissolved with $1 \mathrm{~mL}$ of $0.5 \%$ carboxymethyl cellulose was administered orally by gavage in the $\mathrm{WI}+\mathrm{PFD}$ group, and $1 \mathrm{~mL}$ of $0.5 \%$ carboxymethyl cellulose was administered in both the sham group and the WI group.

All rats were anesthetized with an intraperitoneal injection of sodium pentobarbital $(120 \mathrm{mg} / \mathrm{kg})$. Tracheotomy and mechanical ventilation were performed with a tidal volume of $10 \mathrm{~mL} / \mathrm{kg}$, a respiratory rate of 70 breaths/min, a positive end-expiratory pressure of $2 \mathrm{~cm} \mathrm{H}_{2} \mathrm{O}$, and a fraction of inspiratory oxygen of 1.0. Heparin (50 IU) was injected through the jugular vein, then a left thoracotomy was performed at the fifth intercostal space.

The sham group underwent 210 minutes of perfusion without ischemia. In the WI groups, the left pulmonary hilum was occluded with a vascular clamp to induce WI 30 minutes after pirfenidone or vehicle administration. During clamping of the left hilum, the tidal volume was adjusted to $6 \mathrm{~mL} / \mathrm{kg}$. After 90 minutes of ischemia, the clamp was removed to begin reperfusion for 120 minutes, and the tidal volume recovered to $10 \mathrm{~mL} / \mathrm{kg}$. Details of this procedure are shown in Video 1.

Lung mechanics measurements. After 120 minutes of reperfusion, a median sternotomy was performed. The right pulmonary hilum was occluded with a vascular clamp, and the left lung was ventilated with a tidal volume of $5 \mathrm{~mL} / \mathrm{kg}$ for five minutes. An arterial blood sample was then obtained through the ascending aorta. After that, the rat was connected to a flexiVent (SCIREQ, Montreal, Quebec, Canada) to measure the left pulmonary function. We measured the compliance, mean airway pressure, and peak airway pressure by alternating perturbations of the single forced oscillation families in a closely spaced manner (SnapShot; SCIREQ).

Lung wet-to-dry weight ratio. After measurement of pulmonary function, the left lung was harvested to obtain tissue samples and divided into 3 parts. The upper part was used to calculate the lung wet-to-dry weight ratio. The wet weight was measured soon after harvesting, and the dry weight was measured after 24 hours of desiccation at $100^{\circ} \mathrm{C}$. The ratio was calculated as wet weight divided by dry weight.

Pathologic evaluation. The middle part of the left lung was fixed in $10 \%$ formalin and stained with hematoxylin-eosin. Naphthol AS-D chloroacetate esterase staining was used to count neutrophil infiltration into the perivascular area, as previously reported. ${ }^{15}$ For immunohistochemical staining to detect the apoptotic cells, the avidin-biotin complex method was used. After deparaffinization and blocking with normal bovine serum, the section was incubated with a primary antibody (anti-single strand DNA [ssDNA] antibody) and then with a biotin-labeled secondary antibody in a standard manner. The number of red blood cell was expressed by the average number in 5 randomly chosen high-power fields (HPFs) per section at a magnification of $400 \times$. The number of neutrophils was expressed by the average number in 5 randomly chosen HPFs per section at a magnification of $400 \times$. The number of ssDNA-positive cells, as apoptotic cells, was expressed by the average number in 5 randomly chosen fields at a magnification of $200 \times$. Three separate investigators evaluated in a blinded manner. The degrees of bleeding and pulmonary edema were divided into 4 grades ranging from - to +++ , and the 3 values were averaged.

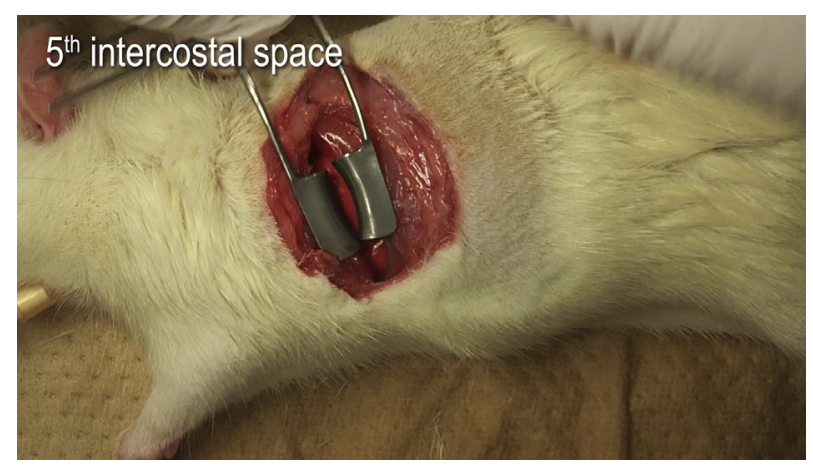

VIDEO 1. Short video showing the procedure of the left hilar clamping in rats and review of this study. Video available at: https://www.jtcvs.org/ article/S0022-5223(18)32493-0/fulltext. 


\section{Quantitative Real-Time PCR}

The lower part of the left lung was obtained for quantitative real time polymerase chain reaction (PCR) to evaluate gene expression of tumor necrosis factor $\alpha$ (TNF- $\alpha$ ) messenger RNA (mRNA), interleukin- $1 \beta$ (IL-1 $\beta$ ) mRNA, myeloperoxidase mRNA, and nuclear factor $\kappa \mathrm{B}$ subunit 1 (NF- $\kappa \mathrm{B}$ ) mRNA. The tissue was stabilized with RNAlater (QIAGEN, Hilden, Germany), and RNA was extracted with an RNeasy Plus Mini Kit (QIAGEN), according to the manufacturer protocol. The total RNA concentration was determined by spectrophotometry, and the quality was evaluated with a 260/280 $\mathrm{nm}$ ratio (1.8-2.0). The total RNA was then reverse transcribed to complementary DNA (cDNA) with Ready-To-Go You-Prime First-Strand Beads (GE Healthcare, Pittsburgh, Pa). Each cDNA was diluted to $10 \mu \mathrm{g} / \mu \mathrm{L}$, and $2 \mu \mathrm{L}$ of cDNA was mixed with the Thunderbird probe qPCR Mix (Toyobo, Osaka, Japan) and TaqMan Gene Expression Assay probe set (Thermo Fisher Scientific, Waltham, Mass). The PCR was performed on the StepOnePlus Real-time PCR system (Thermo Fisher Scientific). The relative quantity was calculated with the comparative $\Delta \Delta \mathrm{C}_{\mathrm{T}}$ method. Glyceraldehyde 3-phosphate dehydrogenase was used as a reference gene and the samples of the sham group were used as controls. Analysis of each sample was run in triplicate. The probes used for PCR were as follows: glyceraldehyde 3-phosphate dehydrogenase (NM_017008), TNF- $\alpha$ mRNA (NM_012675), IL-1 $\beta$ mRNA (NM_031512), myeloperoxidase mRNA (NM_001107036), and NF- $\kappa$ B mRNA (NM_1276711).

\section{Experiment 2}

Concentration of pirfenidone in rat hilar clamp model. Pirfenidone administration $(300 \mathrm{mg} / \mathrm{kg}$ ), 90 minutes of clamping of the left hilum, and 120 minutes of reperfusion were performed as described in experiment $1(\mathrm{n}=10)$.

To confirm the concentration of pirfenidone in the model, the ultraperformance liquid chromatography mass spectrometry method was used. In this experiment, a blood sample was collected through the inferior vena cava, and the lungs were harvested at 30 minutes after pirfenidone administration and 120 minutes after reperfusion ( $\mathrm{n}=5$ for each condition).

Lung weight was measured with an analytic balance (Shimadzu Corp, Kyoto, Japan) before concentration measurement. Next, the lungs were added to saline solution to make a total 10 times the original volume and homogenized with a Polytron PT 1300 D (KINEMATICA AG, Luzern, Switzerland).

For measurement, $50 \mu \mathrm{L}$ of collected plasma and the lung homogenized samples were used, followed by the addition of $100 \mu \mathrm{L}$ of methanol with the internal standard working solution (carbamazepine $500 \mathrm{ng} / \mathrm{mL}$ ). After vortex mixing for 1 minute, the sample was centrifuged at 13,000 rpm for 8 minutes. The supernatant was injected into an ACQUITY UPLC system (Waters Corp, Milford, Mass) for analysis. The standards were prepared in the same way.

The calibration curves were constructed in the concentration range of $5-2,000 \mathrm{ng} / \mathrm{mL}$. Samples exceeding $2000 \mathrm{ng} / \mathrm{mL}$ were measured again with the appropriate dilution.

Chromatographic separation was achieved on an ACQUITY UPLC system with an Acquity BEH C18 column $(2.1 \mathrm{~mm} \times 50 \mathrm{~mm}, 1.7-\mu \mathrm{m}$ particle size Waters Corp) maintained at $30^{\circ} \mathrm{C}$. A gradient program was used with the mobile phase combining solvent $\mathrm{A}(0.1 \%$ formic acid in water) and solvent B (methanol) as follows: $30 \%$ to $90 \%$ B (0-1.9 minutes) and $90 \%$ to $30 \% \mathrm{~B}$ (1.9-2.0 minutes). A 1-minute period of reequilibration was allowed before the next injection. The flow rate was $0.4 \mathrm{~mL} / \mathrm{min}$.

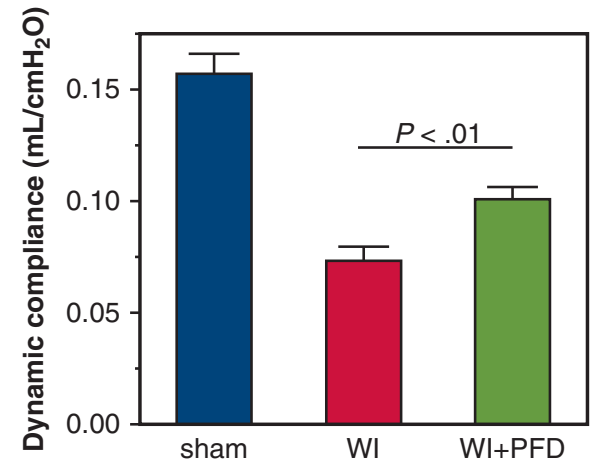

A

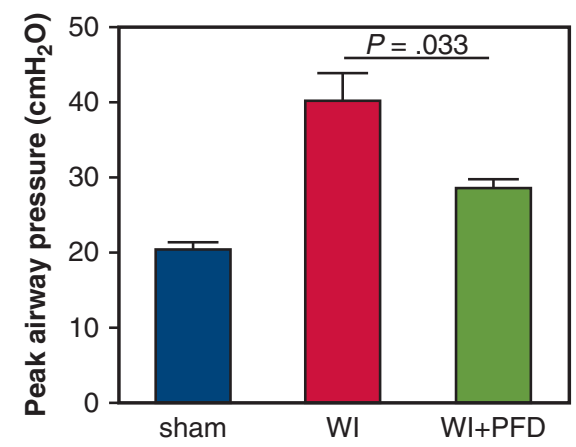

C

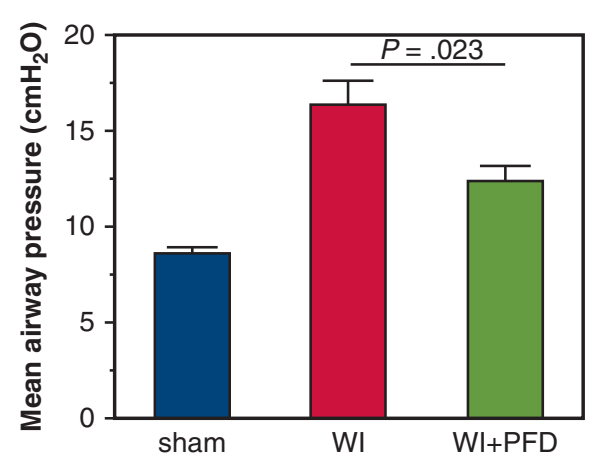

B

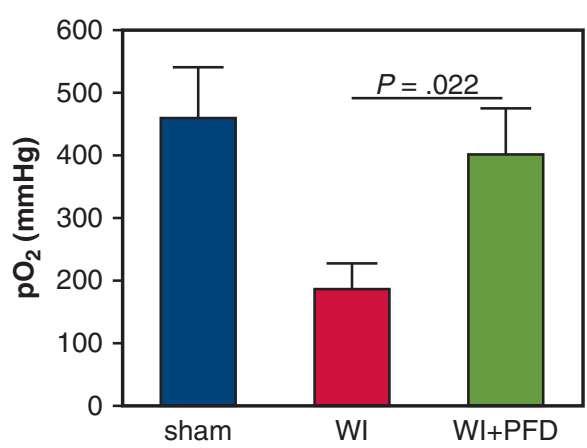

D

FIGURE 1. Pulmonary function of the left lung. A, Dynamic pulmonary compliance. B, Mean airway pressure. C, Peak airway pressure. D, Arterial partial pressure of oxygen. All parameters were significantly improved in the warm ischemia plus pirfenidone $(W I+P F D)$ group relative to the warm ischemia $(W I)$ group. Sham, Sham group. Bars and error bars show mean and standard error of the mean, respectively. 
Pirfenidone and the internal standard were detected with an ACQUITY QDa Detector (Waters Corp) with positive ion electrospray ionization. The detected $\mathrm{m} / \mathrm{z}$ (mass divided by charge number) values were 186.2 for pirfenidone and 237.1 for carbamazepine (internal standard). Data analyses were performed with Empower 3 software (Waters Corp).

\section{Statistical Analysis}

All values are presented as mean \pm standard error of the mean. Analyses were performed with JMP Pro 12.2.0 software (SAS Institute Inc, Cary, NC). One-way analysis of variance was used to compare the means of the 3 groups, and a 2-tailed Dunnett $t$ test was then performed to determine difference. We also performed sensitivity analysis with the Kruskal-Wallis test to confirm the results of the analysis of variance. The correlations between plasma concentration and lung tissue concentration of pirfenidone were calculated with the Pearson product moment correlation analysis.

\section{RESULTS \\ Pulmonary Function of the Left Lung}

The results of the lung function analyses are shown in Figure 1 . Dynamic pulmonary compliance was significantly higher in the WI+PFD group than that in the WI group $(P<.01$; Figure $1, A)$. Mean and peak airway pressures were lower in the WI+PFD group $(P=.023$ and $P=.033$, respectively; Figure $1, B$ and $C$ ). The WI + PFD group had better oxygenation levels $(P=.022$; Figure $1, D)$.

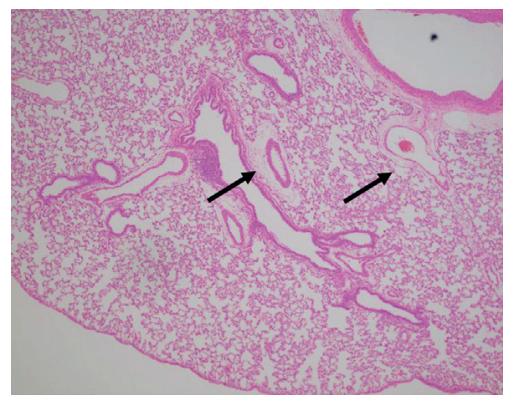

A

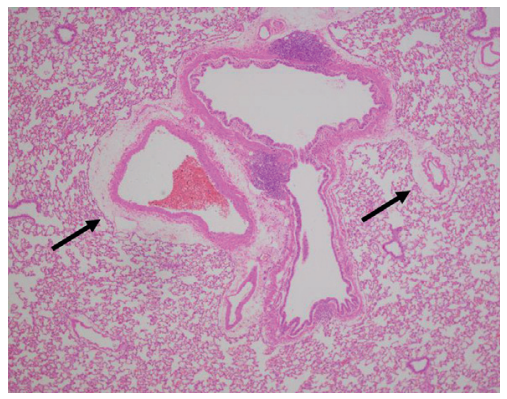

C

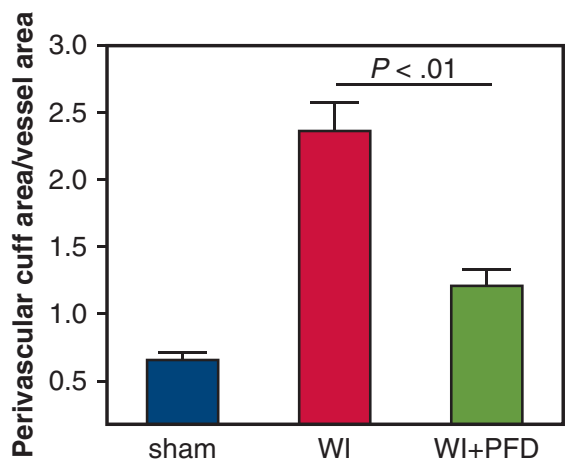

E

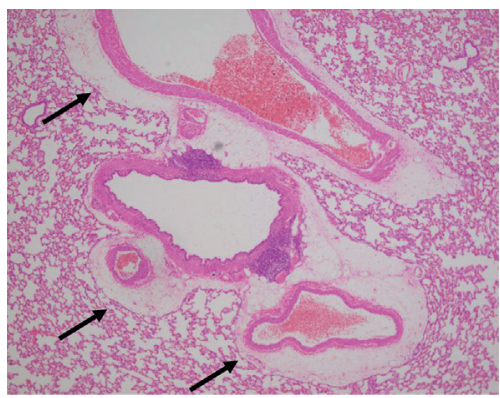

B

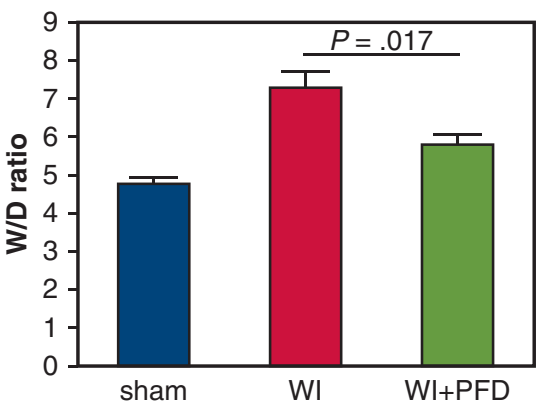

D

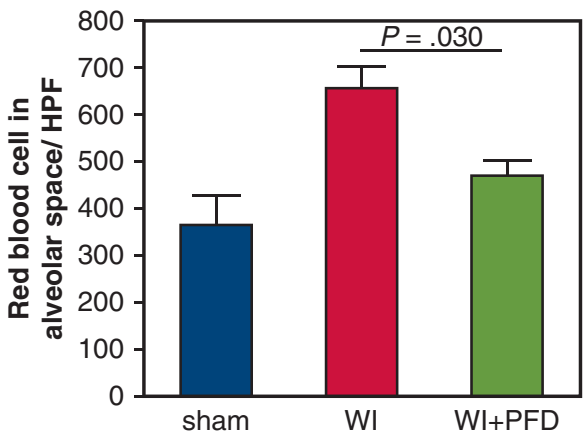

$\mathbf{F}$

FIGURE 2. Hematoxylin and eosin staining and lung wet-to-dry weight $(W / D)$ ratio. A, Sham group. B, Warm ischemia (WI) group. $C$, Warm ischemia plus pirfenidone $(W I+P F D)$ group. D, Wet-to-dry weight ratio. E, The index of perivascular cuff area to vessel area. F, The number of red blood cells in the alveolar space per high-power field $(H P F)$. Arrows indicate perivascular edema. Bars and error bars show mean and standard error of the mean, respectively. 


\section{Histologic Findings}

The left lungs harvested from the sham group showed almost normal histologic results. In contrast, those from the WI group showed severe perivascular edema, alveolar wall swelling, and hemorrhage into the alveolar area. These findings were ameliorated in the WI+PFD group, and the wet-to-dry weight ratio was significantly lower in the $\mathrm{WI}+\mathrm{PFD}$ group than in the WI group $(P=.017$; Figure 2, A-D). We measured the perivascular cuff area in histologic sections of lungs isolated from 5 animals in the sham group (15 vessels), $10 \mathrm{WI}$ animals (30 vessels), and $10 \mathrm{WI}+\mathrm{PFD}$ animals (30 vessels). We calculated the index of perivascular cuff area to the vessel area to eliminate variations related to vessel size. The index was significant lower in the WI+PFD group than in the WI group $(P<.01$; Figure 2, $E)$.

The number of red blood cells in the alveolar space was expressed by the average number in 5 randomly chosen high-power fields (HPFs) per section at a magnification of 400 determined by dynamic cell count (BZ-II Analyzer; Keyence, Osaka, Japan). The number was significant lower in the $\mathrm{WI}+\mathrm{PFD}$ group than in the WI group $(P=.030$; Figure $2, F)$.

The number of neutrophil infiltrations into the perivascular area was markedly increased in the WI group relative to the sham group. Neutrophil infiltration was significantly lower in the WI+PFD group relative to the WI group $(P=.029$; Figure 3$)$.

Immunostaining for ssDNA showed that the number of apoptotic cells was lower in the WI+PFD group than in the WI group $(P=.026$; Figure 4$)$.

\section{Gene Expressions of TNF- $\alpha$, IL-1 $\beta$, and NF- $\kappa$ B in the Lung Tissue}

Gene expression of TNF- $\alpha$ mRNA was significantly reduced in the WI+PFD group relative to the WI group $(P<.05$; Figure 5, A). Although the difference was not significant, the expressions of IL- $1 \beta$ mRNA and myeloperoxidase mRNA were lower in the $\mathrm{WI}+\mathrm{PFD}$ group $(P=.25$ and $P=.19$, respectively; Figure 5, $B$ and $C$ ).

In the WI group, the expression of NF- $\kappa \mathrm{B}$ mRNA was increased 1.7-fold relative to the sham group. In the WI+PFD group, however, the expression was only increased by 1.2-fold relative to the sham group, and there was a significant difference between the WI+PFD group and the WI group $(P=.019$; Figure $5, D)$.

\section{Concentration of Pirfenidone in Plasma and Lung Tissue}

The pirfenidone concentrations in plasma, right lung, and left lung at 30 minutes after administration were $32.9 \pm 10.8 \mu \mathrm{g} / \mathrm{mL}, 34.0 \pm 10.5 \mu \mathrm{g} / \mathrm{g}$ lung weight, and

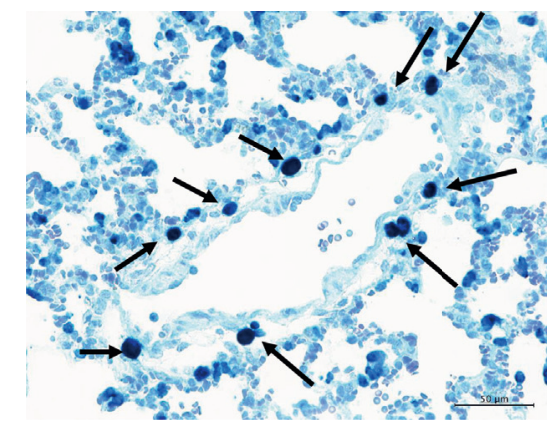

B

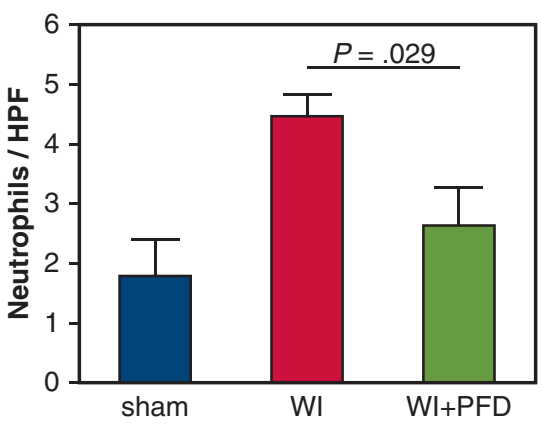

D

FIGURE 3. Naphthol AS-D chloroacetate esterase staining to detect neutrophils. A, Sham group. B, Warm ischemia (WI) group. C, Warm ischemia plus pirfenidone $(W I+P F D)$ group. D, The number of neutrophils in the perivascular area per high-power field $(H P F)$. Neutrophil infiltration $($ arrows) was significantly lower in the warm ischemia plus pirfenidone group relative to the warm ischemia group. Bars and error bars show mean and standard error of the mean. 


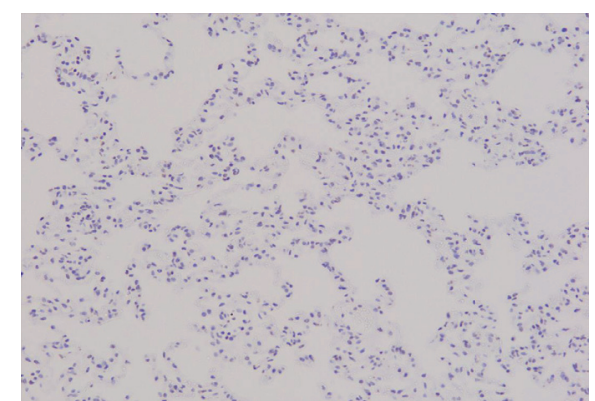

A

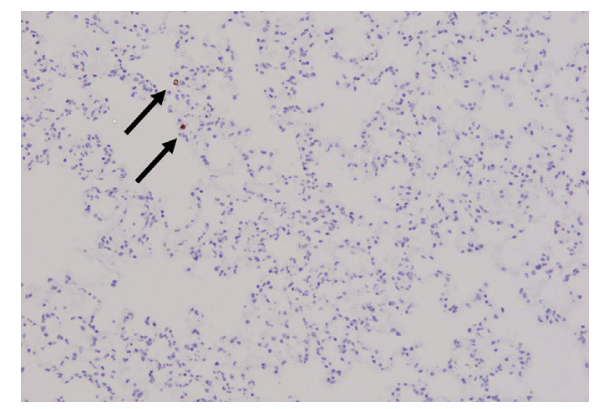

C

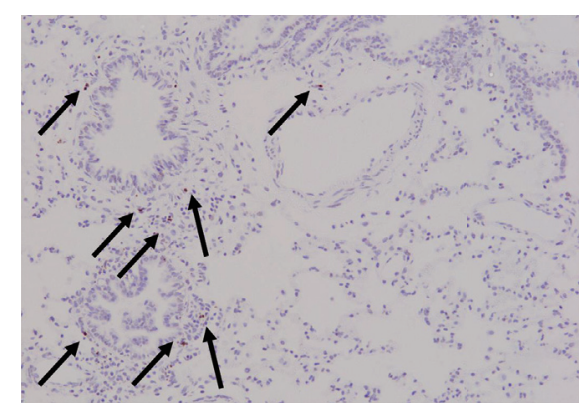

B

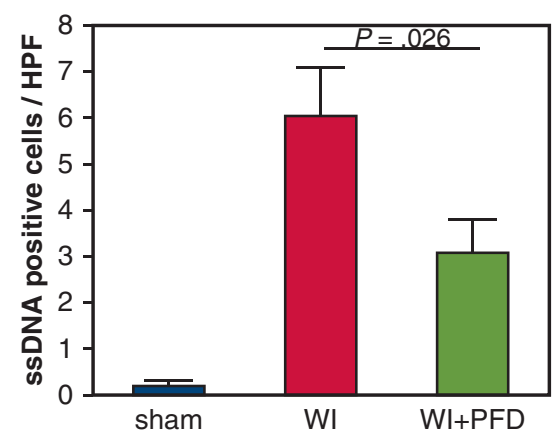

D

FIGURE 4. Immunostaining for single-strand DNA ( $s s D A$ ) to detect apoptotic cells. A, Sham group. B, Warm ischemia group. (C) Warm ischemia plus pirfenidone $(W I+P F D)$ group. (D) The number of single-strand DNA-positive cells per high-power field $(H P F)$. The number of single-strand DNA-positive cells (arrows) was lower in the warm ischemia plus pirfenidone group relative to the warm ischemia group. Bars and error bars show mean and standard error of the mean.

$30.8 \pm 8.60 \mu \mathrm{g} / \mathrm{g}$ lung weight, respectively. At 120 minutes after reperfusion, they were $19.0 \pm 4.32 \mu \mathrm{g} / \mathrm{mL}, 17.3 \pm$ $3.90 \mu \mathrm{g} / \mathrm{g}$ lung weight, and $16.6 \pm 3.02 \mu \mathrm{g} / \mathrm{g}$ lung weight, respectively. The bilateral lung concentrations were strongly correlated with plasma concentrations (right lung, $r=.99, P<.01$; left lung, $r=.98, P<.01)$.

\section{DISCUSSION}

In this study, we demonstrated that administration of pirfenidone in a rat model of lung IRI improved pulmonary function, oxygenation level, pulmonary edema, and neutrophil infiltration. Furthermore, pirfenidone decreased the number of apoptotic cells and proinflammatory cytokines in lung tissue.

There have been some reports that pirfenidone suppresses fibrosis caused by rejection and inflammatory change in the chronic or subacute phase after lung transplantation, ${ }^{16-18}$ but there are no reports of the acute phase, especially within 72 hours, after transplantation. In this study, it was shown that administration of pirfenidone was effective before the onset of lung IRI, which is the main cause of acute lung injury after lung transplantation. Liu and colleagues ${ }^{18}$ reported that pirfenidone inhibits lung fibrosis by decreasing TGF- $\beta$ and arginase activity at 21 days after lung transplantation, but they did not evaluate acute lung injury. In our study, we focused on lung IRI with administration of pirfenidone. The results clearly showed that the lung IRI was alleviated from the acute phase by pirfenidone through inhibition of TNF- $\alpha$ and NF- $\kappa$ B.

In clinical practice, pirfenidone is used for the treatment of IPF. Some studies have reported that it is possible to reduce the incidence of acute exacerbation of IPF associated with surgery by orally administering pirfenidone before and after surgery. ${ }^{19,20}$

The molecular target of pirfenidone has yet to be identified. Previous reports have suggested direct inhibition of TNF- $\alpha$ and reduction of inflammatory cytokines through the NF- $\kappa \mathrm{B}$ pathway. NF- $\kappa \mathrm{B}$ is known as a crucial transcription factor of the inflammatory response. Oxidation by reactive oxygen species causes the release of the inhibitory subunits of the NF- $\kappa \mathrm{B}$ inhibitor and activation of $\mathrm{NF}-\kappa \mathrm{B}$, which generate proinflammatory signaling. ${ }^{21}$ In this study, pirfenidone decreased NF- $\kappa$ B mRNA in the lung tissue as previously reported, and it had a protective effect on acute lung injury. Moreover, $N F-\kappa B$ and TNF- $\alpha$ have an effect on cell survival. ${ }^{22,23}$ This study showed that pirfenidone significantly decreased both of these on lung IRI and reduced apoptotic cells according to the immunohistochemical staining of ssDNA. Staining with anti-ssDNA antibody has been shown 


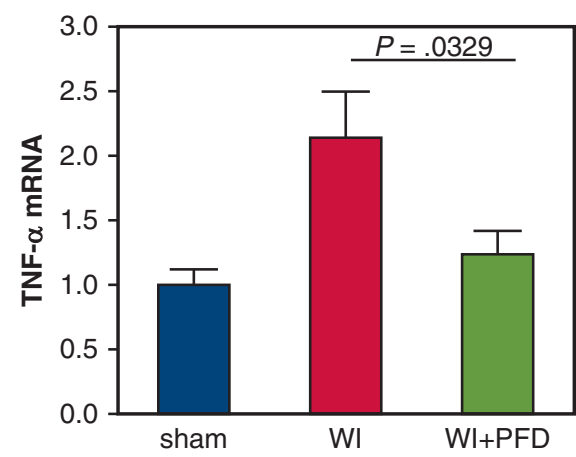

A

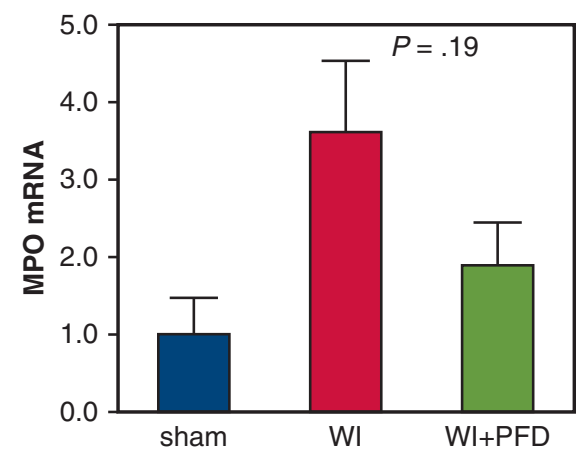

C

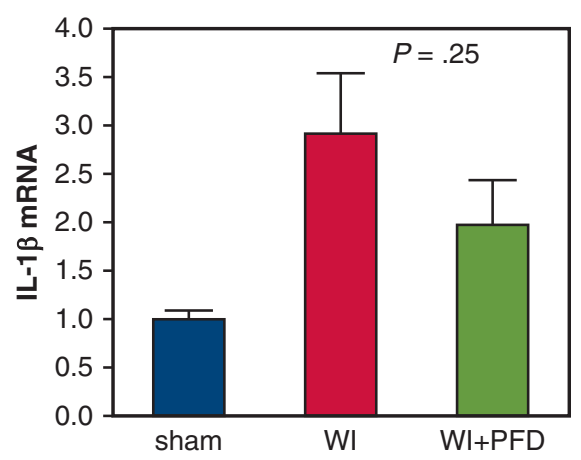

B

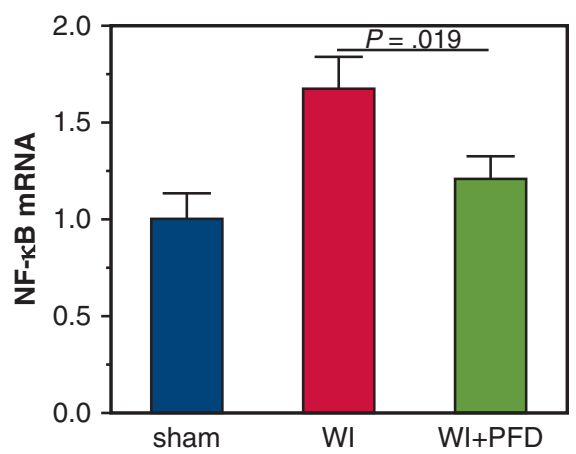

FIGURE 5. Gene expressions in the left lung. A, Tumor necrosis factor- $\alpha(T N F-\alpha)$ messenger RNA ( $m R N A)$. B, Interleukin- $1 \beta$ ( $I L-1 \beta)$ messenger RNA. $\mathrm{C}$, Myeloperoxidase $(M P O)$ messenger RNA. D, Nuclear factor subunit $\kappa \mathrm{B} 1(N F-\kappa B)$ messenger RNA. Gene expression of tumor necrosis factor- $\alpha$ and nuclear factor subunit $\kappa \mathrm{B} 1$ messenger RNA were significantly reduced in the warm ischemia plus pirfenidone $(W I+P F D)$ group relative to the warm ischemia $(W I)$ group.

to be a specific and sensitive procedure for the detection of apoptotic cells relative to terminal deoxynucleotidyl transferase deoxyuridine triphosphate nick end labeling. ${ }^{24}$

In experiment 2, our results demonstrated that administration of pirfenidone orally by gavage in a rat IRI model increased concentrations of pirfenidone in blood and lung tissue within the experimental period. ${ }^{25-27}$

Several limitations of this study should be acknowledged. Pirfenidone had a protective effect on lung IRI in a rat hilar clamp model, but our findings at this stage cannot be directly applied to human lung transplantation. We chose the hilar clamp model in this study for 2 reasons. First, IRI occurs in many clinical settings, and a clamp or vascular occlusion model has been used in the study of IRI in other organs, such as kidney, liver, and intestine. ${ }^{13,14,28-30}$ Second, the hilar clamp model is a simpler and more consistently reproducible procedure than a transplant model. For a proof-of-concept experiment examining pirfenidone, the clamp model was used to limit variability in IRI.

The hilar-clamp model, however, differs from clinical lung transplantation in three aspects. The first is the difference in ischemic condition. In this experiment, reperfusion occurred after WI alone. In contrast, lung transplantation involves a period of cold ischemia followed by WI of the graft. Second, in clinical lung transplantation, there is no pirfenidone present in the allograft before implantation during cold ischemia. Recipients taking pirfenidone before transplant will have lower circulating levels of the drug, given that the last dose might be nearly 24 hours before transplant. The reperfused donor lung will therefore be exposed to zero to low doses of recipient-derived pirfenidone. In this study, it cannot be distinguished whether pirfenidone in the donor lung is effective during ischemia or pirfenidone in the blood or donor lung is effective for the reperfusion. Furthermore, in this study, we had only set a single dose $(300 \mathrm{mg} / \mathrm{kg})$ for the pirfenidone group, but by using presurgical administration and titration of dosage, we could get useful information for clinical use in lung transplantation. In addition, because it is assumed that in lung transplantation, pirfenidone will only be administered to the recipient; it is necessary to consider using the transplant model with administration of to the recipient alone. Third, the response of the immune system of rats is different from that of humans. We need to confirm our findings in other animal models before applying these results to humans.

This was an observational study of the effects of pirfenidone, and we did not clarify the drug's mechanism. 
Further investigation will be necessary to define the mechanism associated with the protective effect of pirfenidone.

In conclusion, pirfenidone administration before ischemia attenuated lung IRI through inhibition of pulmonary edema, production of proinflammatory cytokines, and apoptosis in a rat model. Pirfenidone may be useful as a new option for treatment of lung IRI.

\section{Conflict of Interest Statement}

Pirfenidone was provided by Shionogi \& Co, Ltd. Authors have nothing to disclose with regard to commercial support.

\section{References}

1. Gielis JF, Beckers PA, Briedé JJ, Cos P, Van Schil PE. Oxidative and nitrosative stress during pulmonary ischemia-reperfusion injury: from the lab to the OR. Ann Transl Med. 2017;5:131.

2. Tejwani V, Panchabhai TS, Kotloff RM, Mehta AC. Complications of lung transplantation. a roentgenographic perspective. Chest. 2016;149:1535-45.

3. Chen F, Date H. Update on ischemia-reperfusion injury in lung transplantation. Curr Opin Organ Transplant. 2015;20:515-20.

4. Dang C, Zhai Z, Wu D, Lin Q, Yang Y, Yang M, et al. Inflammatory response and pneumocyte apoptosis during lung ischemia-reperfusion injury in an experimental pulmonary thromboembolism model. J Thromb Thrombolysis. 2015;40:42-53.

5. Pinto A, Immohr MB, Jahn A, Jenke A, Boeken U, Lichtenberg A, et al. The extracellular isoform of superoxide dismutase has a significant impact on cardiovascular ischemia and reperfusion injury during cardiopulmonary bypass. Eur J Cardiothorac Surg. 2016;50:1035-44.

6. Lohser J, Slinger P. Lung injury after one-lung ventilation: a review of the pathophysiologic mechanisms affecting the ventilated and the collapsed lung. Anesth Analg. 2015;121:302-18.

7. Leung CH, Caldarone CA, Wang F, Venkateswaran S, Ailenberg M, Vadasz B, et al. Remote ischemic conditioning prevents lung and liver injury after hemorrhagic shock/resuscitation: potential role of ahumoral plasma factor. Ann Surg. 2015;261:1215-25.

8. Altun GT, Arslantaş MK, Cinel I. Primary graft dysfunction after lung transplantation. Turk J Anaesthesiol Reanim. 2015;43:418-23.

9. Fiser SM, Tribble CG, Long SM, Kaza AK, Kern JA, Jones DR, et al. Ischemia-reperfusion injury after lung transplantation increases risk of late bronchiolitis obliterans syndrome. Ann Thorac Surg. 2002;73:1041-7; discussion 1047-8.

10. Miyorhi R, Chen-Yoshikawa TF, Hijiya K, Motoyama H, Aoyama A, Menju T, et al. Significance of single lung transplantation in the current situation of severe donor shortage in Japan. Gen Thorac Cardiovasc Surg. 2016;64:93-7.

11. Grattendick KJ, Nakashima JM, Feng L, Giri SN, Margolin SB. Effects of three anti-TNF-alpha drugs: etanercept, infliximab and pirfenidone on release of TNF- $\alpha$ in medium and TNF- $\alpha$ associated with the cell in vitro. Int Immunopharmacol. 2008;8:679-87.

12. Castro-Torres RD, Chaparro-Huerta V, Flores-Soto ME, Jave-Suárez L, Camins A, Armendáriz-Borunda J, et al. Pirfenidone attenuates microglial reactivity and reduces inducible nitric oxide synthase mRNA expression after kainic acid-mediated excitotoxicity in pubescent rat hippocampus. J Mol Neurosci. 2015;56:245-54.
13. Arumugam TV, Shiels IA, Margolin SB, Taylor SM, Brown L. Pirfenidone attenuates ischemia-reperfusion injury in the rat small intestine. Clin Exp Pharmacol Physiol. 2002;29:996-1000.

14. Kaibori M, Yanagida H, Uchida Y, Yokoigawa N, Kwon AH, Okumura T, et al. Pirfenidone protects endotoxin-induced liver injury after hepatic ischemia in rats. Transplant Proc. 2004;36:1973-4.

15. Ohsumi A, Chen F, Nakajima D, Sakamoto J, Yamada T, Fujinaga T, et al. Therapeutic effect of surfactant inhalation during warm ischemia in an isolated rat lung perfusion model. Transpl Int. 2012;25:1096-105.

16. Zhou H, Latham CW, Zander DS, Margolin SB, Visner GA. Pirfenidone inhibits obliterative airway disease in mouse tracheal allografts. J Heart Lung Transplant. 2005;24:1557-85.

17. Bizargity P, Liu K, Wang L, Hancock WW, Visner GA. Inhibitory effect of pirfenidone on dendritic cells and lung allograft rejection. Transplantation. 2012;94:114-22.

18. Liu H, Drew P, Gaugler AC, Visner GA. Pirfenidone inhibits inflammatory responses and ameliorates allograft injury in a rat lung transplant model. J Thorac Cardiovasc Surg. 2005; 130:852-8.

19. Iwata T, Yoshida S, Nagato K, Nakajima T, Suzuki H, Tagawa T, et al. Experience with perioperative pirfenidone for lung cancer surgery in patients with idiopathic pulmonary fibrosis. Surg Today. 2015;45:1263-70.

20. Iwata T, Yoshino I, Yoshida S, Ikeda N, Tsuboi M, Asato Y, et al; West Japan Oncology Group. A phase II trial evaluating the efficacy and safety of perioperative pirfenidone for prevention of acute exacerbation of idiopathic pulmonary fibrosis in lung cancer patients undergoing pulmonary resection: West Japan Oncology Group 6711L (PEOPLE Study). Respir Res. 2016;17:90.

21. Sies H, Berndt C, Jones DP. Oxidative stress. Annu Rev Biochem. 2017;86: 715-48.

22. Sun XF, Zhang H. NFKB and NFKBI polymorphisms in relation to susceptibility of tumour and other diseases. Histol Histopathol. 2007;22:1387-98.

23. Borghi A, Verstrepen L, Beyaert R. TRAF2 multitasking in TNF receptor-induced signaling to $\mathrm{NF}-\kappa \mathrm{B}$, MAP kinases and cell death. Biochem Pharmacol. 2016;116:1-10.

24. Frankfurt OS, Robb JA, Sugarbaker EV, Villa L. Monoclonal antibody to single-stranded DNA is a specific and sensitive cellular marker of apoptosis. Exp Cell Res. 1996;226:387-97.

25. Thorat SG, Chikhale RV. Determination and pharmacokinetic study of pirfenidone in rat serum by high-performance thin-layer chromatography. J Chromatogr Sci. 2016;54:1115-9.

26. Sun W, Jiang ZL, Zhou L, Chen RM, Wang Z, Li WS, et al. Determination and pharmacokinetic study of pirfenidone in rat plasma by UPLC-MS/MS. J Chromatogr B Analyt Technol Biomed Life Sci. 2015;981-2:14-8.

27. Togami K, Kanehira Y, Tada H. Pharmacokinetic evaluation of tissue distribution of pirfenidone and its metabolites for idiopathic pulmonary fibrosis therapy. Biopharm Drug Dispos. 2015;36:205-15.

28. Abdelkader A, Ho J, Ow CP, Eppel GA, Rajapakse NW, Schlaich MP, et al. Renal oxygenation in acute renal ischemia-reperfusion injury. Am J Physiol Renal Physiol. 2014;306:F1026-38.

29. Yamamura M, Miyamoto Y, Mitsuno M, Tanaka H, Ryomoto M, Fukui S, et al. Edaravone suppresses reperfusion injury following leg ischemia in rats: a transmission electron microscopic study. Int J Angiol. 2013;22: 267-70.

30. Jennings RB. Historical perspective on the pathology of myocardial ischemia/ reperfusion injury. Circ Res. 2013;113:428-38.

Key Words: ischemia-reperfusion injury, pirfenidone, left hilar clamp model 